\title{
Visualization of Chinese Economy Based on WebGL
}

\author{
Xuezheng $\mathrm{Li}^{1, \mathrm{a}}$ \\ ${ }^{1}$ Scool of Computer Science and Technology, Tianjin University , Tianjin, 300350, China \\ aemail: 18910917386@163.com
}

\begin{abstract}
Keywords: Visualization, Chinese Economy, WebGL; 3D Interaction
\end{abstract}
\begin{abstract}
At present, unbalanced economic development and great regional difference exists in our economy, so it is necessary to study the regional difference of economy. However, financial stock market determines the distribution situation of private capital in regional and inter-industry. This paper has made visualized research on the data of listed companies based on WebGL technology and attained the distribution situation of Chinese capital in different regions and different industries through visual observation and comparative analysis. The tool proposed in this paper plays a guidance role for the country to make economic strategies and decide the direction of individual investment.
\end{abstract}

\section{Introduction}

The grasp of features and rules of economic development stage is the scientific base for one country to make economic strategy and is an important field of making economic theory research, which has aroused continuous and extensive attention of economists all over the world [1]; while the research on regional differences at economic development stage is the field draws important attention of geographer, is an important subject of regional coordination development and is also the basis of making national regional development strategy and regional policies[2]. For China, the following ten years is the crucial period of promoting and realizing coordination development of regional economy; therefore, it needs an objective urgently to clarify the overall strategy of regional development [3].

Temporal and spatial difference of regional economy in China has been an important problem drawing the attention of academic circle all the time. Kanbur $\mathrm{R}$ and others have pointed out that the temporal and spatial distribution of regional economy is the core of the problem, including features of spatial pattern and time evolution[4-5]. Fujita $M$ and others have pointed out that the research scale tends to be diversified and changes from the macroscopic scale of differences between south and north and provincial differences etc to microscopic scale of plain area and coastal area etc[6]. Rozelle $\mathrm{S}$ and others have proposed that exploration of reasons and decisions and suggestions of government have become the main foothold of research[7].

However, there are still some shortages in the research: first, most of research results have not considered about the changes of regional economic differences in time; second, there are big divergences in the indexes or methods taken in the research; third, most of researches are theoretical results, which can't help the decision support of target customer or reason analysis directly.

In view of above analysis, this paper has made visualized analysis of the temporal and spatial distribution of Chinese capital with adoption of visual analysis theory, adopted related data of listed companies in China attained from financial data analysis software Wind and solved the mutual covering problem of large-scale information data on map display with WebGL and other related computer technologies.

\section{Related Work}

At present, visualization of spatial data can be divided into three categories: visualization of point data[8-9], visualization of line data [10] and visualization of regional data 8 . The main method of visualization of point data is to mark the object on the map with various symbols based on its coordinate, but when massive data needs to be marked on the map, a great number of overlapping situations will be produced among points, moreover, uneven data distribution is easily produced, 
which causes the decrease of screen utilization rate. Visualization of line data is to draw lines to link corresponding locations and use lines to express movement trace; massive line data will cause serious visual confusion and it usually takes edge binding technology to make suitable abstraction and aggregation for data. Visualization of regional data is to map statistical data on reach region of map through color but there is asymmetric mapping between data and region, for example, usually massive data concentrates in densely populated regions but these regions are usually with small areas. This paper has adopted Web3D technology to solve the mutually overlapping problem of massive point data sets in geographical space.

The proposing of WebGL technology has solved existing web interactive 3D problem perfectly. WebGL technology standard has eliminated the trouble of developing vray for web page specially and can be used for creating websites with complicated 3D structures and even designing 3D web page games etc. Compared with the realization method of other Web3D, the advantages of WebGL are as following:

- WebGL realizes network interactive 3D animation production through JavaScript. Based on this technology, the client can support 3D image presentation and GPU hardware accelerating without installing plug-ins;

- WebGL is open. At present, Adobe FlashPlayer 11 and Silver light 3.0 have speeded up GPU speed, but they are private and not transparent;

- WebGL makes use of graphic hardware acceleration at bottom layer for graphic rendering and promotes Web developers to display 3D scene and model in browser smoothly with system graphics, at the same time, creates complicated visualization of navigation and data.

Using WebGL programming directly is very complicated. You need to understand the internal details of WebGL and learn complicated shader grammar to use WebGL well. At present, there are a lot of WebGL open source frameworks, such as GLGE, SceneJS and CubicVR etc. Each framework has own different places, but their common target is to construct a senior and friendly WebGL development environment for developer.

The WebGL framework used in this paper is Three.js, which packages the common objects in 3D graphic programming in simple and visual method. Three,js uses a lot of senior skills of graphics engine in development, which has improved the performance greatly. In addition, Three.js is complete open source and can find own source code on github. Three.js covers the details of 3D rendering and abstracts the details of WebGL native API and disassemble 3D scene into grid, material and light source; Three.js is object oriented and developer can use upper layer JavaScript object but not use JavaScript function only; Three.js function is very rich, besides packing WebGL native API, it also includes many practical built-in objects and can be applied in game development and animation production etc; Three.js speed is fast, which adopts 3D graphic best practice to keep extremely high performance under the premise of ensuring not losing usability; Three.js supports interaction and WebGL itself not extracts picking up function but Three.js solidifies picking up support.

\section{Visualization And Realization Of Stock Information}

We attain json data of Chinese map from internet and the data content is as shown in TABLE I. We attain related data of listed companies in China from financial data analysis software Wind and the data content is as shown in TABLE II.

TABLE I. Geographic information data

\begin{tabular}{|l|l|l|l|l|l|}
\hline \multirow{2}{*}{ ID } & \multicolumn{2}{|l|}{ properties } & geometry \\
\cline { 2 - 6 } & name & cp & childNum & type & coordinates \\
\hline xin_jiang & Xin Jiang & {$[84.9023$,} & 18 & Polygon & {$[[96.416,42.75$} \\
& & $41.748]$ & & & $88], \ldots .]$. \\
\hline
\end{tabular}


TABLE II. Geographic information dataStock information data

\begin{tabular}{|c|c|c|l|c|}
\hline company_name & province & city & values & profession \\
\hline Luoyang & & & {$[68535024607.8$} & \\
Luanchuan & Henan & Luoyang & $496, \ldots]$ & Nonferrous \\
Molybdenum & & & metal \\
Group Limited & & & \\
Company & & & \\
\hline
\end{tabular}

In TABLE I, property $\mathrm{cp}$ is the coordinate of provincial capital of one province, which is expressed with latitude and longitude; child Num is the number of cities included in this province; type expresses that whether the border of province is composed of one polygon or several polygons; coordinates is the array of province boundary point, in which each point is expressed with latitude and longitude coordinates. TABLE II needs to explain values property and the representative data is the market value of one company in 13 quarters from Jun. 3rd 2012 to Jun. 3rd 2015. Profession is the belonging industry of this company; company name is the name of the company, province and city represent the province and city that the company belongs to.

Based on above two data sets, we have developed visual analysis system as shown in Figure 1 with WebGL technology. This system includes two parts: the buttons on the left column display the name of each industry and the user can click the buttons of different industries to observe the information of different industries; the right is the main interface and we draw the Chinese map (red part) based on data set as shown in TABLE I. Based on TABLE II, we draw the 3D column chart presenting the sum of company market value of each province; the height of column chart is in right proportion to the sum of company market value of the province and will change with the change of quarter. User clicks the column chart and it will display the pie chart of the proportion of each industry within the province so that user can observe clearly. The user can also click the left buttons and check the performance of different industries.

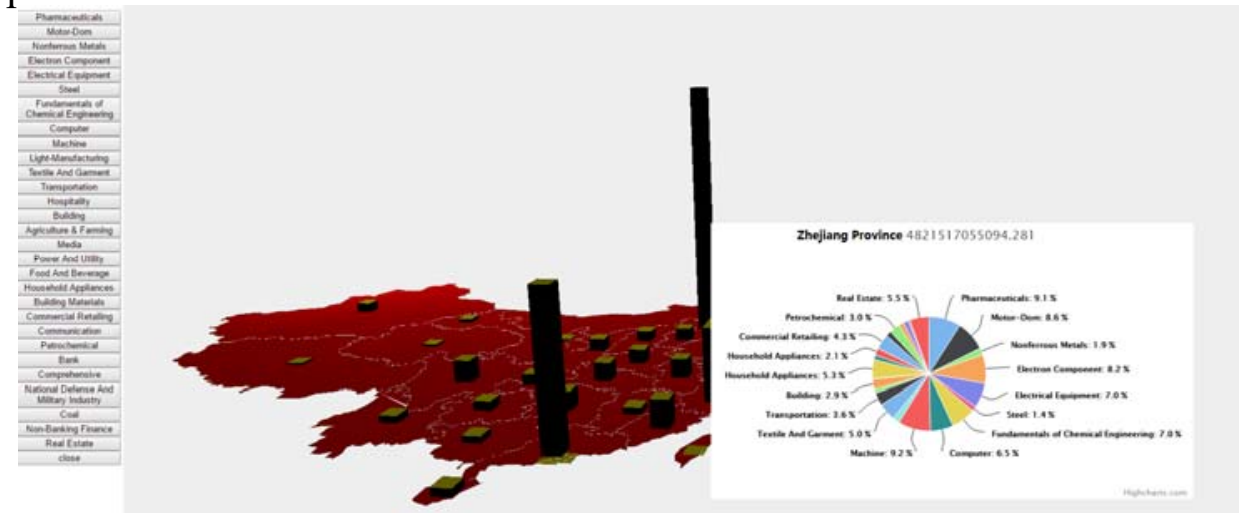

Fig.1. main interface of system

First, we need to solve how to change the latitude and longitude coordinates of map boundary of China into screen coordinates. The definition rule of geological coordinate is increasing to the right at $\mathrm{X}$ axis (represent longitude) and increasing upward on $\mathrm{Y}$ axis (latitude); while the definition rule of screen coordinate is increasing to the right at $X$ axis (represent longitude) and increasing downward on $\mathrm{Y}$ axis (latitude). It can be seen that the only difference between geological coordinate and screen coordinate is that the increasing direction on $\mathrm{Y}$ axis is opposite.

- Known the height $(h)$ and width $(w)$ of screen and the range of geological coordinate area is (maxLon, minLon, maxLat, minLat)

- Calculate the latitude and longitude represented by each pixel (ensure the accuracy, multiply the latitude and longitude of geological coordinates with 3600 to form into seconds):

scaleX $=((\operatorname{maxLon}-$ ninLon $) * 3600) / w$

scaleY $=((\max L a t-n i n L a t) * 3600) / h$

- Calculate the coordinate of any point (lon, lat ) in geological coordinate region on screen:

screen $X=$ lon $* 3600 /$ scaleX

screen $Y=$ lat $* 3600 /$ scale $Y$

- Fill this geological region in the whole image region:

$\min X=$ minLon $* 3600 /$ scaleX 
$\min Y=\operatorname{minLat} * 3600 /$ scale $Y$

$X=\operatorname{screen} X-\min X$

$Y=$ screen $Y-\min Y$

- We finally attain the formula of changing latitude and longitude coordinates into screen coordinates through above deduction process:

$$
\begin{aligned}
& X=(\text { lon }- \text { minLon }) * 3600 / \text { scale } X \\
& Y=(\text { maxLat }- \text { lat }) * 3600 / \text { scale } Y
\end{aligned}
$$

Based on above formula, we change latitude and longitude coordinates in data set into screen coordinates and draw China plane map with WebGL technology.

To realize the target of dynamic change of 3D column chart with the change of time, we include cycle redraw function, with which, we do not render scene in turn but render continuously. There are a lot of methods to realize cycle redraw and one of them is to use setTimeout() callback and reset time lag after rendering of scene finished. However, this method is already outmoded and at present, flow device support a better method requestAnimationFrame(), browser can optimize the performance of animation greatly. Because it will consider about all draw requests and put all of them in one redraw step. Especially in multi-tab browser, when the animation web page is at the backstage, the browser will stop redraw to save the resources and improve performance. We have defined one renderScene() function for cycle redraw, in which we use the render () method to transfer the scene and camera to it, and then we revise the height of $3 \mathrm{D}$ column chart and location property based on the time change to make the column chart move; finally we will transfer this function to requestAnimationFrame() to realize cycle rendering.

WebGL application is a tripod supported by three legs of graph, animation and interaction. To make user analyze the regional economic situation in China in a better way, the interaction function in 3D scene is essential. First, we transfer the mouse coordinates into the coordinates of view port controls (origin is in the center and the coordinates range of $\mathrm{x}$ axis and $\mathrm{y}$ axis is between -0.5 and +0.5 ). Viewpoint coordinates will be transferred into one point in $3 \mathrm{D}$ space coordinates and take this point as origin to emit a ray, starting from this origin and extending to one fixed direction. This pick ray starts from the intersection point of $2 \mathrm{D}$ viewpoint coordinates and near clip plane and points to far clip plane straightly. Any objects intersected with the ray is regarded as at the below of mouse pointer, as shown in Figure 2.

Following things will happen when we click on the screen:

- First, create one vector based on the position we click on screen.

- Second, change the clicking position on screen into coordinates in Three.js scene with unproject function.

- Third, we emit a ray of light to the scene from the clicking position on the screen with THREE.Raycaster object.

- Fourth, we use raycaster.intersectObjects function to judge whether any of the designated objects are shot by this ray of light.

When selecting one 3D column chart, we use highcharts graphics technology and data information represented by column chart to draw the column chart of distribution proportion of various industries, as shown in Figure 3.

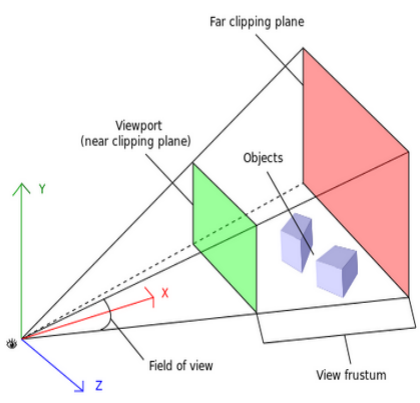

Fig.2. WebGL sketch map

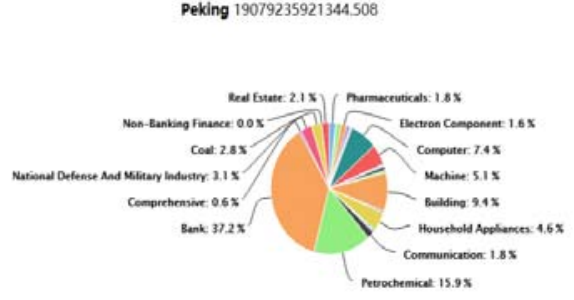

Fig.3. Pie chart of Industry distribution 


\section{Case Study}

We have invited experts in related field of economics at school to use our visualization system. Turn on our application, firstly, it finds out that most of our capitals distribute in Beijing, Shanghai, Guangzhou and Zhejiang, as shown in Figure 4-1; he clicked the column chart representing Beijing and discovered the proportions of various industry capitals, banking and petrochemical industries bear in the brunt, as shown in Figure 4-2; after clicking the left industry buttons, he found out that agriculture, forestry, husbandry and fishery industries distribute evenly within the country, textile and garment industries mainly distribute at coastal areas, especially in Zhejiang, Shanghai and Jiangsu, as shown in Figure 4-3 and 4-4; and then he found some interesting information, building material industry distributes evenly but the building industry focuses in Beijing and real estate industry focuses in Guangzhou, as shown in Figure 4-5, 4-6 and 4-7. In addition, even coal industry is mainly distributed in Beijing, as shown in Figure 4-8.

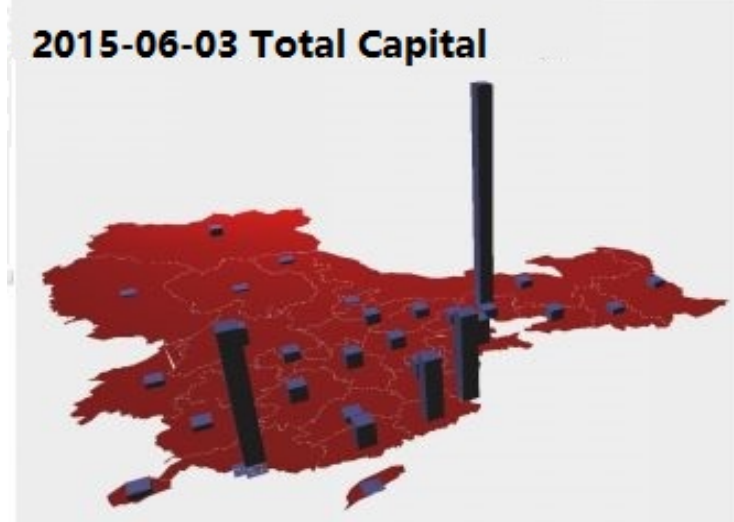

Fig.4-1. Distribution of total capital

2015-06-03 Total Capital

2015-06-03 Agriculture \& Farming

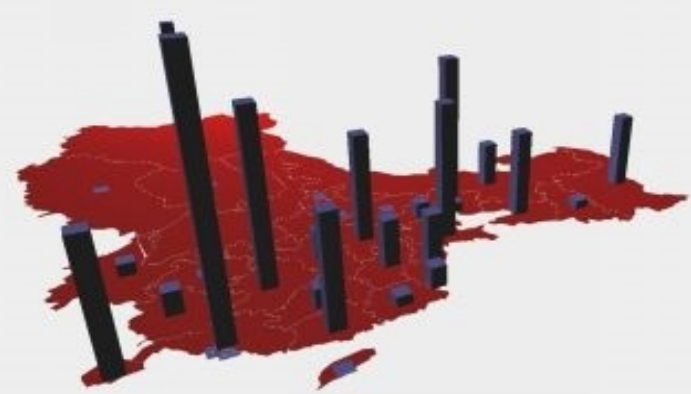

Fig.4-3. Distribution of agriculture \& farming

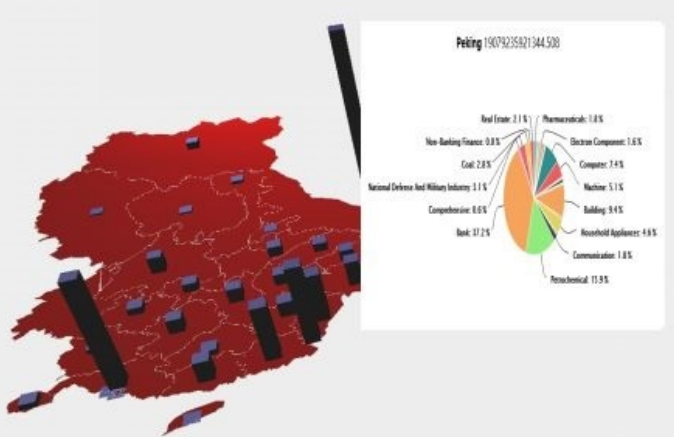

Fig.4-2. Interact with Fig4-1

\section{5-06-03 Textile and garment}

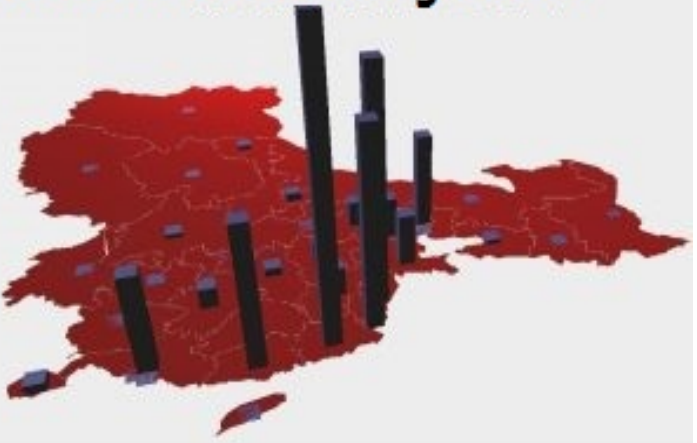

Fig.4-4. Distribution of textile and garment

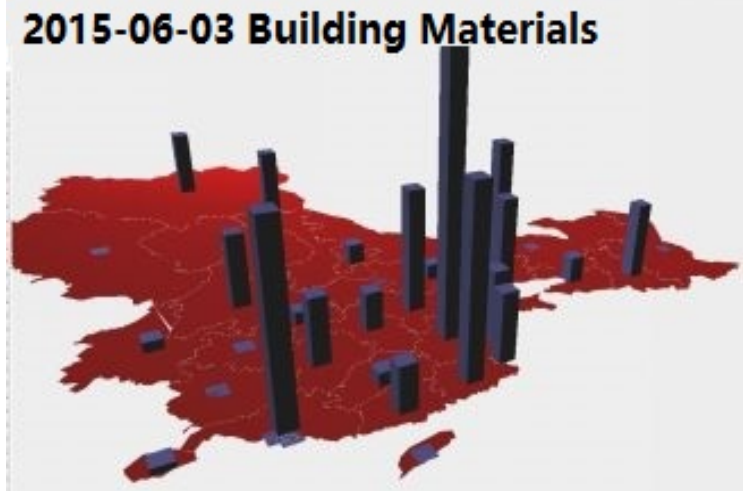

Fig.4-5. Distribution of building materials industry

\section{5-06-03 Building}

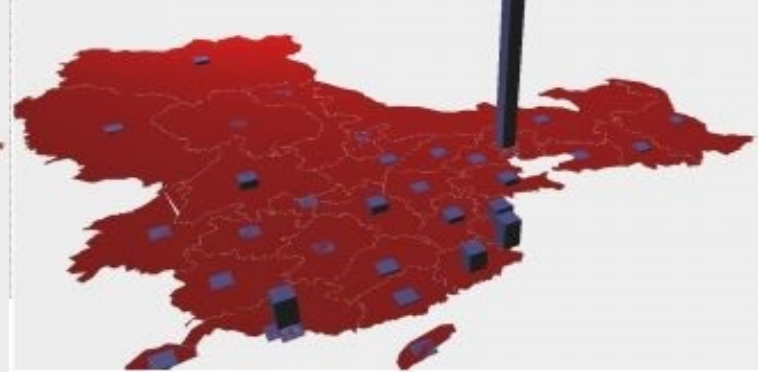

Fig.4-6. Distribution of building industry 


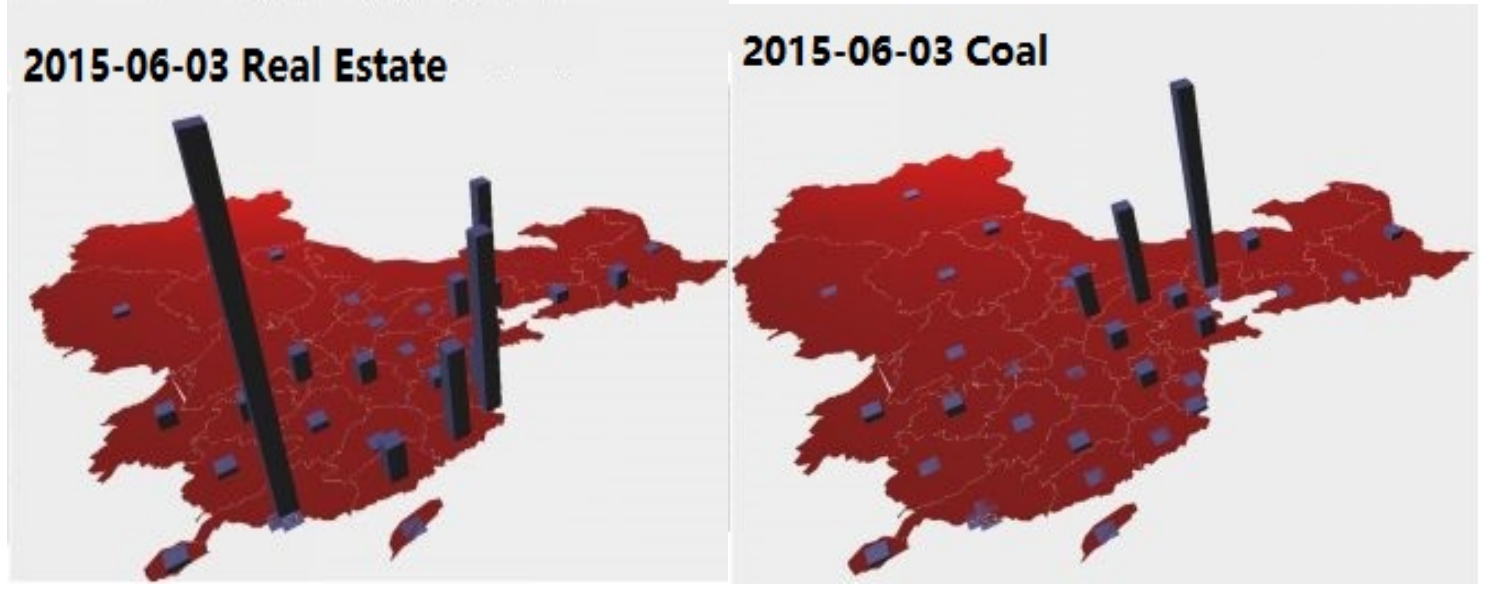

Fig.4-7. Distribution of real estate industry

Fig.4-8. Distribution of Coal industry

\section{Conclusion}

This paper has displayed the distribution of capital at different regions and different industries visually with WebGL technology, presented the industry distribution visually with highcharts and analyzed economic region distribution and reasons on above basis.

The expectations for following work are as following:

- Add into other types of data and realize visualization with capital data.

- Add more intelligent analysis on the basis of visualization.

- Provide more user interactions.

- Optimize the scene graph in WebGL.

\section{References}

[1] Omae K. The end of the nation state: The rise of regional economies[M]. Simon and Schuster, 1995.

[2] Amin A, Thrift N. Globalization, institutions, and regional development in Europe[M]. Oxford university press, 1995.

[3] Wang X S. The Chinese economy in crisis: state capacity and tax reform[M]. Routledge, 2015.

[4] Kanbur R, Zhang X. Which regional inequality? The evolution of rural-urban and inland-coastal inequality in China from 1983 to 1995[J]. Journal of comparative economics, 1999, 27(4): 686-701.

[5] Rey S J, Montouri B D. US regional income convergence: a spatial econometric perspective[J]. Regional studies, 1999, 33(2): 143-156.

[6] Fujita M, Hu D. Regional disparity in China 1985-1994: the effects of globalization and economic liberalization[J]. The Annals of Regional Science, 2001, 35(1): 3-37.

[7] Rozelle S. Rural industrialization and increasing inequality: Emerging patterns in China' s reforming economy

[8] Ward M O, Grinstein G, Keim D. Interactive data visualization: foundations, techniques, and applications[M]. CRC Press, 2010.

[9] Keim D A, Panse C, Sips M, et al. Visual data mining in large geospatial point sets[J]. IEEE Computer Graphics and Applications, 2004, 24(5): 36-44.

[10] Buchin K, Speckmann B, Verbeek K. Flow map layout via spiral trees[J]. IEEE transactions on visualization and computer graphics, 2011, 17(12): 2536-2544. 